\title{
Städte, denkmalgeschützte Anlagen und kulturelles Erbe
}

\author{
Coordinating Lead Authors (CLAs) \\ Alexandra Jiricka-Pürrer \\ Lead Authors (LAs) \\ Alexandra Jiricka-Pürrer, Christiane Brandenburg, Ulrike Pröbstl-Haider \\ Contributing Authors (CAs) \\ Brigitte Allex
}

\subsection{Allgemeine Entwicklung und Trends}

Neben dem Gesundheitstourismus ist auch die Nachfrage nach Städtereisen ${ }^{1}$ in den letzten zehn Jahren im österreichischen Sommertourismus konstant gestiegen (Fleischhacker 2019). Die verstärkte Nachfrage nach Städtereisen spiegelt den Gesamttrend zur kurzfristigen flexibleren Urlaubsplanung (Zellmann und Mayrhofer 2015, 2017) sowie zu mehrmaligen Kurzurlauben (Wirtschaftskammer Tirol 2018; WKO 2018) wider. In der ganzjährigen Betrachtung machen Kultur-, Besichtigungs- und Städteurlaube knapp $10 \%$ der Reisearten in Österreich aus (Statistik Austria 2016, 2017). Für Wien, als größte österreichische Stadtdestination, zeigen die Ankünfte einen positiven Trend in den letzten zehn Jahren (MA 23 Dezernat Statistik Wien 2018a). Bei den Herkunftsmärkten ist Deutschland das wichtigste Land. Bei den Gästen aus dem Inland, die den zweitwichtigsten Herkunftsmarkt für den Wientourismus darstellen, zeigen die aktuellsten Statistiken aus 2018 ein deutliches Plus von $6 \%$ bei den Nächtigungen im Vergleich zu 2017. Mit größerem Abstand finden sich danach die USA als Herkunftsmarkt auf Platz drei für Wienreisen (MA 23 Dezernat Statistik Wien 2018b)

Auch in Innsbruck und Salzburg sind die Nächtigungszahlen im Sommerhalbjahr in den letzten zehn Jahren stetig gestiegen. So wurden in Innsbruck 2009 noch ca. 571.000 Nächtigungen verzeichnet und 2018 bereits etwa 855.000 (Stadt Innsbruck 2019a). In Salzburg wurden 2014 etwas mehr als 8 Mio. Nächtigungen verzeichnet, 2019 waren es ca. 10,7 Mio., allein zwischen Mai und August (Land Salzburg 2019). Hinsichtlich der Quellmärkte unterscheiden sich

\footnotetext{
${ }^{1}$ Hierunter sind Reisen nach Wien und die Landeshauptstädte zu verstehen. Der Besuch von Kleinstädten wird im vorliegenden Bericht dagegen als eine Ergänzung zu anderen Urlaubsformen bzw. Aktivitäten gesehen, die in den entsprechenden Kapiteln behandelt werden.
}

die beiden Städte jedoch von Wien. In Innsbruck sind inländische Gäste der wichtigste Quellmarkt, gefolgt von Deutschland und Italien. Die USA nehmen hier Platz vier bei den Quellmärkten ein (Stadt Innsbruck 2019b).

Für Städtetourismus in ganz Österreich zeigt der ITRTourismusreport (Fleischhacker 2019) einen Zuwachs von $3,6 \%$ in der Sommersaison 2018. Hinter dem Gesundheitstourismus (mit 3,7 \% Steigerung) ist dies der stärkste $\mathrm{Zu}$ wachs aller Tourismuskategorien bei den Nächtigungen im Sommer 2018 gewesen (Fleischhacker 2019).

Eines der Hauptmotive von Städtereisen ist „Kultur“ erleben. Betrachtet man touristische Statistiken verschiedener Destinationen bzw. Quellmärkte in bzw. für Österreich, so machen Kulturinteressierte im Schnitt rund $12 \%$ aller Gäste aus (RMB 2008; Oberösterreich Tourismus 2015). Auch bei den „Urlaubsarten im Sommer“ haben Kultururlaube gemäß WKO (2018) einen Anteil von $12 \%$ der Gesamturlaube in Österreich in der Sommersaison. Die Forschungsgemeinschaft Urlaub und Reisen (FUR 2017) zeigt, dass die Zahlen für den deutschen Quellmarkt vergleichbar sind - bei den „Kurzurlaubsreisen 2016“ hatten die Kulturreisen ebenfalls einen Anteil von $12 \%$. Bei Städtereisenden nimmt das Motiv „Sehenswürdigkeiten und Kultur“ einen besonders hohen Stellenwert ein. Für den Besuch in Wien gaben $74 \%$ der Gäste „Sehenswürdigkeiten \& Kultur“ als Entscheidungsgrundlage für ihre Reise an (Wien Tourismus 2019).

\subsection{Relevante Entwicklungen in den wichtigsten Herkunftsländern}

Blickt man auf die wichtigsten Herkunftsländer für den Städtetourismus, insbesondere für Wien, dann könnte, in Abhängigkeit von den Einreisebedingungen, der Brexit Auswirkungen haben. Bezogen auf Italien und Spanien, die 
ebenfalls wichtige Herkunftsländer für den österreichischen Städtetourismus darstellen, ist unklar, ob Hitzewellen in den Heimatländern Einfluss auf eine verstärkte Reisetätigkeit in alpine bzw. nördlicher gelegene Länder haben werden. Im Kontext der internationalen Publikationen ist hier jedoch nur eine vorsichtige Erwartungshaltung angebracht (siehe Abschn. 9.3). Weitere wichtige Quellmärkte für den österreichischen Städtetourismus sind China, Russland und im Sommer auch die Vereinigten Arabischen Emirate (Wien Tourismus 2018). Insbesondere bei Gästen aus China (und anderen asiatischen Ländern) wird noch ein Zuwachs erwartet, wenngleich der chinesische Quellmarkt sehr heterogen ist (Thuen Jørgensen et al. 2017). Durch die vergleichsweise kurze Aufenthaltsdauer asiatischer Gäste ist ihr Beitrag zur Wertschöpfung in den einzelnen Städtedestinationen deutlich geringer im Vergleich zu anderen Quellmärkten. Jedoch im Sinne des Klimaschutzes ist eine Förderung der zwei Hauptquellmärkte - Deutschland und Österreich - sowie weiterer europäischer Quellmärkte zu favorisieren, insbesondere bei gleichzeitigem Ausbau bzw. der intensivierten Förderung und Vermarktung der öffentlichen Anreise.

\subsection{Einfluss des Klimawandels auf den Städtetourismus bzw. Einfluss auf den Klimawandel}

\subsubsection{Einflüsse des Klimawandels}

Für österreichische Städte könnten, je nach Lage und Topografie, insbesondere Starkregenereignisse, Stürme und Hitzewellen vermehrt im touristischen Kontext relevant werden. Für mittelgroße und große Städte ist Hitze das am meisten diskutierte Thema in Zusammenhang mit möglichen Anpassungspotenzialen (Fleischhacker und Formayer 2007; Kromp-Kolb et al. 2007; Fleischhacker et al. 2009; Götz et al. 2012; Stadt Wien 2016; Steininger et al. 2016). Hier zeigt sich ein heterogenes Bild zwischen der empfundenen Belastung der städtischen Bevölkerung und jener der Gäste.

So zeigen aktuelle Untersuchungen aus Graz, Leibnitz (Babcicky und Seebauer 2016) und Wien (Juschten et al. 2019a, b), dass die Wohnbevölkerung bereits erschwerte Lebens- und Arbeitsbedingungen durch längere Hitzeperioden für sich wahrnimmt. Die Befragung der Wiener Bevölkerung im Rahmen der REFRESH-Studie zeigt, dass knapp die Hälfte der Befragten die warmen Temperaturen ihrer Stadtwohnung zwar noch als ,erträglich“ empfindet, etwas mehr als ein Drittel der Befragten Hitze aber bereits als belastend wahrnimmt. Für den Hitzesommer 2015 haben rund $45 \%$ angegeben, dass sie die Hitze als „,belastend“ und „nachts schwierig" empfunden haben (Juschten et al. 2019a).
Zahlreiche internationale Studien haben in den letzten zehn Jahren den Einfluss des Wetters bzw. der klimatischen Bedingungen auf die Buchungsentscheidung untersucht (Krajasits et al. 2008; Serquet und Rebetez 2011; Dubois et al. 2016; FUR 2017; Gómez-Martín et al. 2017; Zellmann und Mayrhofer 2017).

Dubois et al. (2016) zeigen für die französische Stichprobe, dass generell angekündigte Hitzewellen weniger Einfluss auf Änderungen der Reiseentscheidung haben als angekündigte Schlechtwetter- und Kälteperioden. Auch retrospektiv ist die Erinnerung an Regenperioden - insbesondere bei längeren Reisen - deutlich negativer besetzt als jene an Hitzewellen am Urlaubsort. Gössling et al. (2016) untersuchten, wie komplex die Einstufung des Reisewetters als „extrem“ ist und wie unterschiedlich der längerfristige Effekt der Erinnerung daran ist. Serquet und Rebetez (2011) sowie Dubois et al. (2016) zeigen hierbei, dass eine hohe „Hitzetoleranz“ bei Reisenden besteht. Im Vergleich zu anderen Reiseformen ist bei Städtereisen die akzeptable Temperatur (bevor sie von Touristinnen und Touristen als ,zu heiß“ empfunden wird) höher als beispielsweise bei Aufenthalten in Berggebieten.

Auch die bisherigen Entwicklungen für den Wientourismus zeigen bislang keine negativen Effekte der Hitzeproblematik. Sie belegen vielmehr einen kontinuierlichen Trend hin zur stärksten Auslastung in den Sommermonaten Juli und August (Wien Tourismus 2018). Im Hitzesommer 2018 hatte der Städtetourismus in ganz Österreich den stärksten Nächtigungszuwachs aller Reisekategorien der Sommersaison nach dem Gesundheitstourismus (Fleischhacker 2019). Auch wenn die fünf wärmsten Sommer der letzten zehn Jahre (2008-2018) betrachtet werden, zeigt sich ein starkes Plus beim Städtetourismus (stärkstes Nachfrageplus aller Sommertourismuskategorien; Fleischhacker 2019). Somit ist bisher kein Hinweis eines negativen Einflusses durch eine mögliche Hitzebelastung für den österreichischen Städtetourismus in der tatsächlichen, statistisch belegten Nachfrage gegeben.

Allex et al. (2011a) zeigten jedoch in ihrer Befragung Wiener Touristinnen und Touristen, dass bereits vor knapp zehn Jahren 32,6 \% der befragten Personen ihr Programm aufgrund von Hitze adaptiert haben. Dabei gab es eine Verschiebung der Aktivitäten, indem bewusst längere Pausen eingeplant oder kühlere Orte aufgesucht wurden. Signifikante Unterschiede gab es vor allem bei längeren Aufenthalten, in diesem Fall wurde deutlich häufiger das Programm angepasst. Abb. 9.1 zeigt die Unterschiede, wie Städtetouristinnen und -touristen die Lokalitäten hinsichtlich der Hitzebelastung generell bewerteten.

Somit kann nicht ausgeschlossen werden, dass sich die Hitzeproblematik zukünftig auf das Buchungsverhalten auswirken wird (z. B. hinsichtlich der gebuchten Jahreszeit oder der Aufenthaltsdauer) bzw. dass entsprechende Effekte zwar bereits bestehen, aber durch das grundsätzliche Wachstum des Städtetourismus verschleiert werden. 
Abb. 9.1 Temperaturempfinden der Befragten bei bzw. in Sehenswürdigkeiten, Einkaufsstraßen/Shoppingcentern sowie Lokalen/Restaurants; $n=365$. (Allex et al. 2011a, S. 19)
Empfanden Sie die hohen Temperaturen als störend?

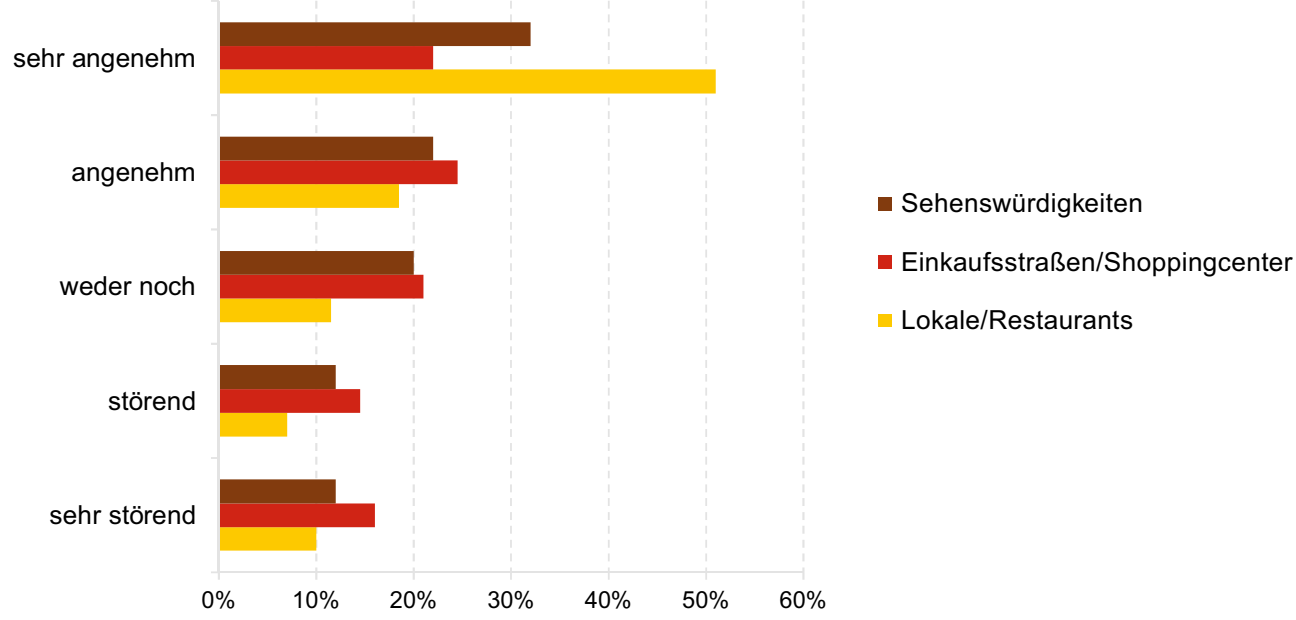

\subsubsection{Einflüsse auf den Klimawandel}

Je nach Herkunftsmarkt erfolgt die Anreise in Österreichs Städte per Flugzeug über die Flughäfen Wien, München oder Innsbruck und in Kombination mit dem Schienenfernverkehr in die anderen städtischen Destinationen (vgl. Abschn. 3.1.1). Quellmärkte für den österreichischen Städtetourismus sind - neben Deutschland - die USA, China, Russland und im Sommer auch die Vereinigten Arabischen Emirate (siehe Abschn. 9.2). Diese interkontinentalen Flüge wirken sich, insbesondere ohne Kompensation, negativ auf das globale Klima aus und sind in Kombination mit der Gesamtaufenthaltsdauer in Europa und der Verkehrsmittelwahl innerhalb des Kontinents der primär ausschlaggebende Faktor in der $\mathrm{CO}_{2}$-Bilanz von Städtereisen.

Betrachtet man die meist kurze Aufenthaltsdauer im Vergleich zu anderen Reisearten und geht man von wiederholten Reiseaktivitäten zu städtischen Destinationen innerhalb eines Jahres aus, so haben auch die innereuropäischen Reiseaktivitäten von Städtereisenden eine negative Auswirkung auf den Klimawandel, sofern sie als Flug- oder Pkw-Reisen durchgeführt werden. Anreize mit Sparpreisen im Zugverkehr wirken dem entgegen. Im Gegensatz zu anderen Reisearten ist jedoch der Transport vor Ort überwiegend klimaverträglich aufgrund der öffentlichen Verkehrsangebote in städtischen Destinationen (vgl. Abschn. 3.3). Hier ergeben sich auch Potenziale, eine öffentliche Anreise weiter zu fördern, da keine zusätzliche Ausrüstung (wie z. B. im Wintersport notwendig) transportiert werden muss und der ÖV schon mehrheitlich für die „letzte Meile“ genutzt werden kann. Während die städtische Infrastruktur im Rahmen der Angebotsvielfalt von Städtereisenden mitgenutzt wird, kommt der Unterkunft im Blick auf die Klimabilanz eine große Bedeutung zu. In diesem Zusammenhang spielt vor allem die Kühlung gemein- sam mit der Anpassung an die Hitze eine zunehmend stärkere Rolle in der Klimabilanz (siehe Abschn. 9.4). Im Vergleich zu anderen europäischen Destinationen hat die österreichische Städtehotellerie hier noch Aufholbedarf.

Möglich wäre auch eine Anpassung bei der Wahl des Quartiers, indem in durchgrünte Außenbezirke bzw. ins Stadtumland ausgewichen wird. Bisher gibt es dazu jedoch noch keine konkreten Untersuchungen. Auch in diesem Zusammenhang sind wiederum die aktive Bewerbung und Unterstützung des klimaschonenden Transfers ins Stadtzentrum eine wichtige Planungsaufgabe.

\subsection{Anpassungsstrategien und Anpassungsbeispiele}

Wenngleich ein Einfluss der Hitze auf den Städtetourismus in Österreich erst vereinzelt nachgewiesen wurde (siehe Abschn. 9.3), wurde in den letzten Jahren in zahlreichen $\mathrm{Pu}-$ blikationen der Hitzestress in Städten als ein vordergründiges Problem mit starkem Anpassungsbedarf und starker Handlungsrelevanz seitens der Stadtplanung hervorgehoben. So widmet beispielsweise die „Österreichische Strategie zur Anpassung an den Klimawandel" (BMNT 2017) den Grünund Freiflächen in den Städten ein eigenes Kapitel inklusive Handlungsempfehlungen. Die Stadt Wien hat sich speziell mit dem Thema ,städtische Hitzeinseln“ auseinandergesetzt. Ergebnis ist ein Strategieplan, in dem Maßnahmen gegen eine zunehmende Erwärmung des Stadtgebietes aufgezeigt werden (Brandenburg et al. 2012; Allex et al. 2013). Ähnliche Maßnahmen empfiehlt das Bundesamt für Umwelt in der Schweiz (BAFU 2018). Weitere Vorteile zur Reduktion von Hitzeinseln können außerdem durch entsprechende Freiflächen - Green Infrastructure (EC 2016) - entstehen, die 


\begin{tabular}{|c|c|}
\hline $\begin{array}{l}\text { Maßnahmen, mit welchen sich die hohen } \\
\text { Temperaturen in Wien angenehmer gestalten lassen }\end{array}$ & SUMME \\
\hline Nichts notwendig/keine & 406 \\
\hline Mehr/gratis/günstige Trinkmöglichkeiten & 144 \\
\hline Klimaanlagen in Hotels, Lokalen, Geschäften ... & 97 \\
\hline Mehr Beschattung & 54 \\
\hline Sprühnebel & 27 \\
\hline Mehr/bessere Information & 21 \\
\hline Mehr/gepflegteres Grün/Plätze & 20 \\
\hline Schwimmbäder/gratis Schwimmen für eine Stunde & 16 \\
\hline Angepasstes (Alternativ-)Programm/Pausen & 16 \\
\hline Ventilatoren (in Hotels, Lokalen, Geschäften) & 13 \\
\hline Kleidung anpassen & 9 \\
\hline Wasser zum Abkühlen & 7 \\
\hline Mehr Sitzgelegenheiten & 6 \\
\hline Sonnen-/Hitzeschutzutensilien & 6 \\
\hline Angepasste Öffnungs-/Betriebszeiten & 5 \\
\hline Mehr Lokale/Lokale der "anderen Art" & 4 \\
\hline Mehr (Freiluft-)Veranstaltungen & 3 \\
\hline Gratis Eis & 3 \\
\hline Offener Bus für Touristen & 1 \\
\hline Mehr Toiletten & 1 \\
\hline Segway-/Fahrradstationen & 1 \\
\hline Nette Gesellschaft & 1 \\
\hline Kein schwarzer Straßenbelag & 1 \\
\hline
\end{tabular}

Abb. 9.2 Maßnahmen gegen die hohen Temperaturen in Wien (Mehrfachantworten - offene Fragen, $n=361$ ). (Verändert nach Allex et al. 2011a, S. 24)

Niederschläge aufnehmen und den Wasserabfluss verlangsamen (Rückhaltefunktion auch bei Starkregenereignissen; Zebisch et al. 2018).

Vorrangig betreffen diese Maßnahmen die lokale Bevölkerung, es können sich jedoch auch positive Synergien für den Städtetourismus ergeben. Der Erhalt bzw. die Aufwertung von Grünräumen und deren vielfältige Nutzungsmöglichkeiten, die Schaffung von wasserbezogenen Freiraumstrukturen sowie wassergebundene Sport- und Erholungsangebote und - soweit möglich - wasserbezogene Mobilität können wertvolle Bausteine für die touristische Angebotsgestaltung im Städtetourismus im Sommer darstellen.

Der Anpassungsbedarf an Hitzewellen in der Hotellerie bzw. in der Gastronomie wird in den Kap. 4 und 5 ausführlicher betrachtet. Durch die, im Vergleich zu anderen Ländern, in manchen Beherbergungskategorien noch sehr geringe Ausstattung mit Kühlungsanlagen kommt einer klimaverträglichen Kühlung eine große Bedeutung zu, um Maladaptation zu vermeiden (BMWFJ 2010; Allex et al. 2011a; BMWFW et al. 2015). Abb. 9.2 zeigt die starke Relevanz von Gebäudekühlung aus der Sicht der Gäste. Neben der Gebäudekühlung ist die Bereitstellung von Trinkwasser wichtig. Die Stadt Wien hat daher bereits entsprechende Maßnahmen getroffen und Trinkwasserbrunnen installiert (Allex et al. 2011b).

\subsection{Handlungsoptionen, Kommunikations- und Forschungsbedarf}

\subsubsection{Ansatzpunkte für Maßnahmen, die Überwindung von Barrieren und Kooperation}

Im Städtetourismus besteht ein wesentlicher Unterschied, ob die Stadt selbst der Zielort der Reise ist oder eine von mehreren Attraktionen des nahegelegenen Urlaubsorts (z. B. Schlechtwetterprogramm für ländlichen Tourismus oder Tagesdestination einer Rundreise zu Kultur und Kulinarik). Reisende, die gezielt einen Städteurlaub in eine städtische Destination planen, könnten vermehrt über Arrangements, die eine klimaverträgliche Anreise und Unterkunft kombinieren, angesprochen werden. Vereinzelt werden bereits Anreize zur klimaschonenden Anreise in der städtischen Hotellerie gesetzt (z. B. Rabatte auf Nächtigungspreise bei Vorlage eines Zugtickets bzw. Nachweis einer anderen klimaverträglichen Anreisemodalität).

In diesem Zusammenhang sind sowohl Kooperationen von Verkehrsbetrieben mit der Hotellerie als auch mit der Tourismuswerbung wichtig. Insbesondere für Geschäftsreisende, die einen wesentlichen Anteil der Städtereisen ausmachen, ist ein verstärktes Attraktivieren der klimaverträglichen Anreise eine wichtige Herausforderung in Ergänzung zu den bereits bestehenden Initiativen zur Förderung und Vermarktung einer klimaschonenden Mobilität in der städtischen Destination (z. B. Kongresstickets für den ÖV). Da Geschäftsreisende selbst ihre Reiseaktivitäten primär nach Gesichtspunkten der Zweckmäßigkeit ausrichten, ist hier vermehrte Bewusstseinsbildung durch Kongressveranstaltende bzw. Unternehmen, die Green Events ausrichten notwendig (vgl. Kap. 10).

\subsubsection{Ansatzpunkte für die Akzeptanz von Maßnahmen, Information und Bewusstseinsbildung}

Klimaverträgliche Bauweisen bei Unterkünften sind in Kombination mit Design und innovativen Nutzungsmöglichkeiten der Gebäudestrukturen für einzelne Zielgruppen interessant. Die Anpassungsmöglichkeit und -kapazität an Hitze wird im städtischen Tourismus vor allem interdisziplinär zu analysieren und weiterzuentwickeln sein, da starke Überschneidungen mit Entscheidungen der Städteplanung, insbesondere der Freiraumplanung, bestehen. Verbesserungen werden auch durch eine touristische Planung für städtische Destinationen erwartet, die bei der Maßnahmenentwicklung auch überregionale touristische Ziele miteinbezieht, um beispielsweise Angebote mit kühleren Attraktionen in Tagesdistanz zu integrieren und zu bewerben, wie beispielsweise Schutzgebiete, Berggebiete, aber auch kulturelle Attraktionen wie Klöster. Auswirkungen 
anderer wetterbedingter Extremereignisse (Starkregenereignisse, Stürme, Nassschnee etc.) betreffen für österreichische Städte vor allem den Transport, insbesondere die An- und Abreise. Hierbei sind die Empfehlungen des Kap. 3 relevant. Wie bereits in Abschn. 9.3 erwähnt, können Maßnahmen zur Anpassung an Hitze bzw. zur Reduktion von urbanen Hitzeinseln auch für die Anpassung an Starkregenereignisse förderlich sein.

\subsubsection{Wissenslücken und Forschungsbedarf}

Auch wenn bisherige Statistiken zeigen, dass insbesondere die Hitzebelastung wenig Einfluss auf das Buchungsverhalten hatte, so ist der zukünftige Einfluss von Extremereignissen auf den Städtetourismus in Österreich bislang kaum erforscht worden. Zusammenhänge zwischen Hitzetoleranz und den Bedürfnissen und Motiven der Zielgruppen, aber auch der Einfluss von städteplanerischen Parametern (z. B. des Grünflächenanteils) wurden bisher im Kontext des österreichischen Städtetourismus ebenfalls wenig betrachtet. Wichtige Aspekte sind dabei die Erreichbarkeit und Zugänglichkeit der grünen und blauen Infrastruktur ${ }^{2}$ sowie die (noch) akzeptierte Distanz zu selbigen (oder zu alternativen, gekühlten Indoorattraktionen) je nach Zielgruppe. Hierbei wäre auch eine Untersuchung der unterschiedlichen Akzeptanz je nach Quellmarkt wichtig.

Weiterhin könnten sich Besichtigungsrouten verlagern, wie von Allex et al. (2011a) erstmals nachgewiesen wurde, da die akzeptable fußläufige Distanz zwischen Sehenswürdigkeiten bei großer Hitze abnimmt. Hier ist einerseits die Städteplanung vermehrt gefordert, Hitzeinseln entgegenzuwirken und verstärkt Schattenbereiche im Freiraum zu schaffen bzw. ggf. zusätzliche Kühlung der Freiflächen zu ermöglichen. Alternativ ist die Kapazität der klimatisierten ÖV-Verbindungen zwischen Sehenswürdigkeiten zu prüfen, da sich die Nachfrage der Nutzung von einzelnen Linien erhöhen könnte. Gegebenenfalls sind höhere Frequenzen in die Mobilitätsplanung miteinzubeziehen. Konkrete, aktuelle Studien für die österreichischen Städtedestinationen (insbesondere Wien und Graz mit starker, zunehmender Hitzebelastung) zur Akzeptanz der Maßnahmen bzw. Veränderungen im touristischen Tagesprogramm können wichtige Hinweise für die Vermarktung und Produktgestaltung bieten.

\subsection{Zusammenfassung}

Anpassungsoptionen für den Städtetourismus ergeben sich primär durch die Zunahme von Hitzewellen und ihre Auswirkungen auf die Verstärkung städtischer Hitzeinseln (hohe

\footnotetext{
2 Unter grüner und blauer Infrastruktur versteht man die Ausstattung mit Grünflächen, Parks und Freiflächen an Gewässern.
}

Übereinstimmung, starke Beweislage). Synergien für den Tourismus ergeben sich durch die städtebaulichen bzw. freiraumplanerischen Anpassungsziele, die insbesondere eine Begrünung der dichter bebauten Bereiche forcieren ebenso wie eine Kühlung von großen Plätzen (hohe Übereinstimmung, starke Beweislage). Größere Grünareale sowie Wasserflächen könnten vermehrt in die touristische Angebotsgestaltung eingebunden werden ebenso wie kühlere Indoorangebote (beispielsweise Besuche von Kulturinstitutionen, Kirchen u. a.). Entsprechend ist ihre öffentliche Anbindung, aber auch die Wahrung der Interessen der städtischen Bevölkerung zu beachten (hohe Übereinstimmung, mittlere Beweislage).

Für den Klimaschutz ist die Reduktion des Flugverkehrs bei der Anreise bedeutsam. Sowohl außereuropäische Gäste auf Rundreisen als auch Städtereisende aus dem stärksten Quellmarkt Deutschland könnten durch Anreize und Marketinganstrengungen zu einer verstärkten Nutzung von Hochgeschwindigkeitszügen animiert werden (hohe Übereinstimmung, starke Beweislage).

\section{Kernaussagen - Kapitel 9}

- Je nach Größe und Lage der städtischen Destination sind Hitze bzw. Hitzewellen und deren Auswirkungen die größte Herausforderung in den nächsten Jahrzehnten für den Städtetourismus (hohe Übereinstimmung, starke Beweislage).

- In Hinblick auf den Klimaschutz zählen die Förderung der klimaschonenden Anreise (Reduktion des hohen Anteils an Flugreisen) sowie die Reduktion des Energieverbrauchs in der Beherbergung im Vordergrund (insbesondere um dem stärkeren Energiebedarf durch höheren Kühlungsbedarf in den Sommermonaten entgegenzuwirken) zu den wichtigsten Aufgabenfeldern (hohe Übereinstimmung, starke Beweislage).

- Anpassungsmaßnahmen, die im Rahmen der Stadtentwicklung (z. B. zur Reduktion von Hitzeinseln) geplant und durchgeführt werden, sind auch für die touristische Anpassung und Angebotsentwicklung von hoher Bedeutung und schaffen Synergieeffekte. Verstärkte Kommunikation und Austausch zwischen Stadtplanung und Tourismus sind wichtige Voraussetzungen, um Co-Benefits zu erzielen und Lock-inEffekte zu vermeiden. Dabei ist auch die Berücksichtigung der Verkehrsplanung bzw. eine Abstimmung mit den Verkehrsbetrieben zu beachten (hohe Übereinstimmung, starke Beweislage). 


\section{Literatur}

Allex, B., Brandenburg, C., Liebl, U., Gerersdorfer, T. \& Czachs, C. (2013) Hot Town, Summer in the City - Entwicklung von hitzerelevanten Anpassungsstrategien im Städtetourismus. In: Schrenk, M., Popovich V., Zeile, P. \& Elisei, P. (Hrsg.) Proceedings of the $18^{\text {th }}$ International Conference on Urban Planning, Regional Development and Information Society (REAL CORP 2013), S. 393-398. CORP (Competence Center of Urban and Regional Planning), SchwechatRannersdorf, Österreich. Online unter: https://conference.corp.at/ fileadmin/proceedings/CORP2013_proceedings.pdf (letzter Zugriff: 24.05.2020).

Allex, B., Liebl, U., Brandenburg, C., Gerersdorfer, T. \& Czachs, C. (2011a) Hot town, summer in the city: die Auswirkungen von Hitzetagen auf das Freizeit- und Erholungsverhalten sowie das Besichtigungsprogramm von StädtetouristInnen - dargestellt am Beispiel Wiens. Endbericht von StartClim2010.F in StartClim2010: Anpassung an den Klimawandel - Weitere Beiträge zur Erstellung einer Anpassungsstrategie für Österreich. Online unter: http://www.startclim.at/fileadmin/user_upload/StartClim2010_reports/StCl10F_mitAnhang.pdf (letzter Zugriff: 01.06.2020).

Allex, B., Liebl, U., Brandenburg, C., Gerersdorfer, T. \& Czachs, C. (2011b) ,Hot town, summer in the city“: die Herausforderungen vermehrter Hitzetage im Städtetourismus, Management Letter. Bundesministerium für Wissenschaft, Forschung und Wirtschaf (BMWFW), Wien, Österreich. Online unter: https://www.wko.at/branchen/tourismus-freizeitwirtschaft/hotellerie/minimiert_Kern_und_Umschlag Management_Letter_2_2_12.pdf (letzter Zugriff: 01.06.2020).

Babcicky, P. \& Seebauer, S. (2016) PATCH:ES Private Adaptation to Climate Change: Fallstudienbericht Klimawandelanpassung von Privathaushalten. Wegener Center für Klima und Globalen Wandel, Universität Graz, Österreich. Online unter: http://anpassung.ccca. at/patches/wp-content/uploads/sites/2/2017/04/1-1_PATCHES_Fallstudienbericht_Privathaushalte_BabcickySeebauer_compressed.pdf (letzter Zugriff: 01.06.2020).

BAFU (2018) Hitze in Städten: Grundlagen für eine klimagerechte Stadtentwicklung. Bundesamt für Umwelt (BAFU), Bern, Schweiz. Online unter: https://www.are.admin.ch/dam/are/de/dokumente/agglomerationspolitik/publikationen/hitze-in-staedten-de.pdf.download.pdf/Hitze_in_Staedten_de.pdf(letzter Zugriff: 01.06.2020).

BMNT (2017) Die österreichische Strategie zur Anpassung an den Klimawandel. Aktualisierte Fassung, Jänner 2017. Bundesministerium für Nachhaltigkeit und Tourismus (BMNT), Wien, Österreich. Online unter: https://www.bmlrt.gv.at/umwelt/klimaschutz/klimapolitik_national/anpassungsstrategie/strategie-kontext.html (letzter Zugriff: 28.04.2020).

BMWFJ (2010) Neue Wege im Tourismus:die neue österreichische Tourismusstrategie. Bundesministerium für Wirtschaft, Familie und Jugend (BMWFJ), Wien, Österreich. Online unter: https://www. bmlrt.gv.at/dam/jcr:7d0389dd-4ae8-4726-b254-b88b90da00f0/ Strategie_Neue\%20Wege\%20im\%20Tourismus.pdf (letzter Zugriff: $01.06 .20 \overline{2} 0)$

BMWFW, WKO \& ÖHV (2015) Energiemanagement in der Hotellerie und Gastronomie (Leitfaden, 3. überarbeitete Auflage). Bundesministerium für Wissenschaft, Forschung und Wirtschaft (BMWFW), Wirtschaftskammer Österreich (WKO) und Österreichische Hoteliervereinigung (ÖHV), Wien, Österreich. Online unter: https://www. wko.at/branchen/tourismus-freizeitwirtschaft/hotellerie/Energiemanagement-in-Hotellerie-und-Gastronomie.pdf (letzter Zugriff: 28.05.2020)

Brandenburg, C., Allex, B., Liebl, U. \& Czachs, C. (2012) Hitze und deren Auswirkungen auf den Städtetourismus - dargestellt am Beispiel Wiens. In: Mayer, H. (Hrsg.) Berichte des Meteorologischen Instituts der Albert-Ludwigs-Universität Freiburg Nr. 23, S. 92. Albert-Ludwigs-Universität Freiburg, Deutschland. Online unter: https://freidok.uni-freiburg.de/dnb/download/15274 (letzter Zugriff: 01.06.2020).

Dubois, G., Ceron, J.-P., Gössling, S. \& Hall, C.M. (2016) Weather preferences of French tourists: lessons for climate change impact assessment. Climatic Change 136(2), 339-351. DOI: https://doi. org/10.1007/s10584-016-1620-6

EC (2016) Supporting the implementation of green infrastructure: $f$ nal report. European Commission (EC), Directorate-General for the Environment und Trinomics B.V., Rotterdam, Niederlande. Online unter: https://ec.europa.eu/environment/nature/ecosystems/docs/ green_infrastructures/GI\%20Final\%20Report.pdf (letzter Zugriff: 01.06.2020).

Fleischhacker, V. (2019) Der Sommertourismus in Österreich 2018: Tendenzen der Nachfragesegmente. ITR - Institut für touristische Raumplanung Ges.m.b.H., Tulln an der Donau, Österreich.

Fleischhacker, V. \& Formayer, H. (2007) Die Sensitivität des Sommertourismus in Österreich auf den Klimawandel. Endbericht von StartClim2006.D1 in StartClim2006: Klimawandel und Gesundheit, Tourismus, Energie. Online unter: http://www.startclim.at/fileadmin/ user_upload/reports/StC106D1.pdf (letzter Zugriff: 24.05.2020).

Fleischhacker, E., Formayer, H., Seisser, O., Wolf-Eberl, S. \& KrompKolb, H. (2009) Auswirkungen des Klimawandels auf das künftige Reiseverhalten im österreichischen Tourismus am Beispiel einer repräsentativen Befragung der österreichischen Urlaubsreisenden. BOKU-Met Report 19. Im Auftrag des Bundesministeriums für Wirtschaft, Familie und Jugend (BMWFJ), Wien, Österreich. Online unter: https://meteo.boku.ac.at/report/ (letzter Zugriff: 05.05.2020).

FUR (2017) ReiseAnalyse 2017: erste ausgewählte Ergebnisse der 47. Reiseanalyse zur ITB 2017. FUR Forschungsgemeinschaft Urlaub und Reisen e. V., Kiel, Deutschland. Online unter: https://reiseanalyse.de/wp-content/uploads/2017/09/RA2017_Erste_Ergebnisse DE.pdf (letzter Zugriff: 01.06.2020).

Gómez-Martín, M.B., Armesto-López, X.A. \& Martínez-Ibarra, E. (2017) Tourists, weather and climate: official tourism promotion websites as a source of information. Atmosphere 8(12), 255. DOI: https://doi.org/10.3390/atmos8120255

Gössling, S., Abegg, B. \& Steiger, R. (2016) "It was raining all the time!": ex post tourist weather perceptions. Atmosphere 7(1), 10. DOI: https://doi.org/10.3390/atmos7010010

Götz, A., Burkhardt, A., Manser, R., Marendaz, E., Willi, H.P., Hohmann, R., Köllner-Heck, P. \& Probst, T. (2012) Anpassung an den Klimawandel in der Schweiz: Ziele, Herausforderungen und Handlungsfelder. Erster Teil der Strategie des Bundesrates vom 2. März 2012. Bundesamt für Umwelt (BAFU), Bern, Schweiz. Online unter: https://www.bafu.admin.ch/dam/bafu/de/dokumente/klima/ ud-umwelt-diverses/anpassung_an_denklimawandelinderschweiz. pdf.download.pdf/anpassung_an_denklimawandelinderschweiz.pdf (letzter Zugriff: 01.06.2020).

Juschten, M., Brandenburg, C., Hössinger, R., Liebl, U., Offenzeller, M., Prutsch, A., Unbehaun, W., Weber, F. \& Jiricka-Pürrer, A. (2019a) Out of the city heat - way to less or more sustainable futures? Sustainability 11(1), 214. DOI: https://doi.org/10.3390/su11010214

Juschten, M., Jiricka-Pürrer, A., Unbehauen, W. \& Hössinger R. (2019b) The mountains are calling! An extended TPB model for understanding metropolitan residents' intentions to visit nearby alpine destinations in summer. Tourism Management 75, 293-306. DOI: https:// doi.org/10.1016/j.tourman.2019.05.014

Krajasits, C., Andel, A., Neugebauer, W., Stanzer, G., Wach, I., Kroisleitner, C. \& Schöner, W. (2008) ALSO WIKI: alpiner Sommertourismus in Österreich und mögliche Wirkungen des Klimawandels. Endbericht von Start-Clim2007.F in StartClim2007: Auswirkungen des Klimawandels auf Österreich, Fallbeispiele. Online unter: http:// www.startclim.at/fileadmin/user_upload/reports/StCl07F.pdf (letzter Zugriff: 01.06.2020).

Kromp-Kolb, H., Formayer, H. \& Clementschitsch, L. (2007) Auswirkungen des Klimawandels auf Wien unter besonderer Berücksichti- 
gung von Klimaszenarien. Im Auftrag der Klimaschutzkoordinationsstelle Wien. Institut für Meteorologie und Physik, Universität für Bodenkultur Wien, Österreich. Online unter: https://www.accc.gv.at/ pdf/klimawandel-wien.pdf (letzter Zugriff: 01.06.2020).

Land Salzburg (2019) Landesstatistik: endgültige Monatsdaten, Jahresüberblick 2019. Land Salzburg, Österreich. Online unter: https:// www.salzburg.gv.at/statistik_Documents/statistik-Tourismus-bisherigesKalenderjahr2019.pdf (letzter Zugriff: 01.06.2020).

MA 23 Dezernat Statistik Wien (2018a) Gästeankünfte in Wien nach Herkunftsländern 2007 bis 2017. Stadt Wien, Österreich. Online unter: https://www.wien.gv.at/statistik/wirtschaft/tabellen/ankuenftelaender-zr.html (letzter Zugriff: 21.11.2019).

MA 23 Dezernat Statistik Wien (2018b) Nächtigungen in Wien (Top 10) 1-12/2018. WienTourismus, Wien, Österreich. Online unter: https://b2b.wien.info/de/presse/bilanz2018\#grafiken (letzter Zugriff: 11.02.2019).

Oberösterreich Tourismus (2015) Der Oberösterreich-Gast im Sommer: Ergebnisse der Gästebefragung Tourismus Monitor Austria Sommer 2014. Oberösterreich Tourismus, Linz, Österreich. Online unter: https://www.oberoesterreich-tourismus.at/uploads/media/TMONA_Sommer_gesamt_2014.pdf (letzter Zugriff: 01.06.2020).

RMB (2008) Leitfaden: neue Wege im Kulturtourismus. Regionalmanagement Burgenland GmbH (RMB), Pinkafeld, Österreich. Online unter: http://www.kreativwirtschaft.net/media/file/832_Leitfaden Kulturtourismus.pdf (letzter Zugriff: 01.06.2020).

Serquet, G. \& Rebetez, M. (2011) Relationship between tourism demand in the Swiss Alps and hot summer air temperatures associated with climate change. Climatic Change 108(1-2), 291-300. DOI: https:// doi.org/10.1007/s10584-010-0012-6

Stadt Innsbruck (2019a) Ankünfte und Übernachtungen nach Beherbergungsarten in den Sommerhalbjahren 1986-2019. Landeshauptstadt Innsbruck, Österreich. Online unter: https://www.innsbruck. gv.at/data.cfm?vpath=redaktion/ma_i/allgemeine_servicedienste/ statistik/dokumente38/tourismus3/tourismusshjinsgljrpdf (letzter Zugriff: 15.11.2019).

Stadt Innsbruck (2019b) Übernachtungen in den gewerblichen Beherbergungsbetrieben nach ausgewählten Herkunftsländern. Landeshauptstadt Innsbruck, Österreich. Online unter: https://www. innsbruck.gv.at/data.cfm?vpath=redaktion/ma_i/allgemeine_servicedienste/statistik/dokumente38/tourismus3/tjuebernachtungennationengewerblljrpdf (letzter Zugriff: 15.11.2019).

Stadt Wien (2016) Klimatologische Kenntage in Wien 1954 bis 2016. Stadt Wien, Österreich. Online unter: https://www.wien.gv.at/statistik/lebensraum/tabellen/eis-hitze-tage-zr.html (letzter Zugriff: 22.01.2020).

Statistik Austria (2016) Tourismus; Ankünfte, Übernachtungen, Urlaubsreisen. In: Bundesanstalt Statistik Österreich (Hrsg.) Statistisches Jahrbuch 2016, S. 420-429. Bundesanstalt Statistik Österreich, Wien, Österreich.
Statistik Austria (2017) Mehr als 4 Mio. Auslandsurlaube im Sommer 2016 (Pressemitteilung: 11.565-125/17). Bundesanstalt Statistik Österreich, Wien, Österreich. Online unter: http://www.statistik. at/wcm/idc/idcplg?IdcService=GET_PDF_FILE\&RevisionSelectionMethod $=$ LatestReleased\&dDocName $=\overline{1} 13103$ (letzter Zugriff: 01.06.2020).

Steininger, K.W., Bednar-Friedl, B., Formayer, H. \& König, M. (2016) Consistent economic cross-sectoral climate change impact scenario analysis: method and application to Austria. Climate Services 1, 39-52. DOI: https://doi.org/10.1016/j.cliser.2016.02.003

Thuen Jørgensen, M., Law, R. \& King., B.E. (2017) Understanding the past, anticipating the future - a critical assessment of China outbound tourism research. Journal of Travel \& Tourism Marketing 34(7), 880-891. DOI: https://doi.org/10.1080/10548408.2016.1220889

Wien Tourismus (2018) Ankünfte Wien 2003-17. Wiener Tourismusverband, Wien, Österreich. Online unter: https://b2b.wien.info/de/statistik/daten/ankuenfte-naechtigungen (letzter Zugriff: 11.02.2019).

Wien Tourismus (2019) Der Wiener Urlaubsgast (Private Urlaube in entgeltlichen Unterkünften 2017/2018): Wiener Gästebefragung 2017/2018im Rahmen des Tourismus Monitors Austria (T-MONA). Wiener Tourismusverband, Wien, Österreich. Online unter: https:// b2b.wien.info/media/files-b2b/t-mona-ergebnisse.pdf/download (letzter Zugriff: 20.11.2019).

Wirtschaftskammer Tirol (2018) Tourismus: die Sommerfrische ist wieder da. Wirtschaftskammer Tirol, Innsbruck, Österreich. Online unter: https:/www.wirtschaft.tirol/2017/11/29/sommertourismusdie-frische-ist-wieder-da/ (letzter Zugriff: 22.01.2020).

WKO (2018) Tourismus und Freizeitwirtschaft in Zahlen: österreichische und internationale Tourismus- und Wirtschaftsdaten, 54. Ausgabe, Juni 2018. Wirtschaftskammer Österreich (WKO), Bundessparte Tourismus und Freizeitwirtschaft, Wien, Österreich. Online unter: https://www.wko.at/branchen/tourismus-freizeitwirtschaft/ tourismus-freizeitwirtschaft-in-zahlen-2018.pdf (letzter Zugriff: 28.05.2020).

Zebisch, M., Vaccaro, R., Niedrist, G., Schneiderbauer, S., Streifeneder, T., Weiß, M., Troi, A., Renner, K., Pedoth, L., Baumgartner, B. \& Bergonzi, V. (Hrsg.) (2018) Klimareport Südtirol 2018. Eurac Research, Bozen, Italien. Online unter: http:/www.eurac.edu/de/research/mountains/remsen/projects/Documents/klimareport/Klimareport\%202018\%20DE_new.pdf (letzter Zugriff: 01.06.2020).

Zellmann, P. \& Mayrhofer, S. (2017) Wann Urlaubsreisen gebucht werden ... Der Countdown für den Sommerurlaub läuft. ift Forschungstelegramm 4/2017. IFT Institut für Freizeit- und Tourismusforschung, Wien, Österreich. Online unter: https://www. freizeitforschung.at/data/forschungsarchiv/2017/162.\%20FT\%20 4-2017_Urlaubsbuchung.pdf (letzter Zugriff: 01.06.2020).

Zellmann, P. \& Mayrhofer, S. (2015) Die Urlaubsrepublik: die Zukunft des Tourismus in Österreich. MANZ, Wien, Österreich.

Open Access Dieses Buch wird unter der Creative Commons Namensnennung 4.0 International Lizenz (http://creativecommons.org/licenses/by/4.0/deed.de) veröffentlicht, welche die Nutzung, Vervielfältigung, Bearbeitung, Verbreitung und Wiedergabe in jeglichem Medium und Format erlaubt, sofern Sie den/die ursprünglichen Autor(en) und die Quelle ordnungsgemäß nennen, einen Link zur Creative Commons Lizenz beifügen und angeben, ob Änderungen vorgenommen wurden.

Die in diesem Buch enthaltenen Bilder und sonstiges Drittmaterial unterliegen ebenfalls der genannten Creative Commons Lizenz, sofern sich aus der Abbildungslegende nichts anderes ergibt. Sofern das betreffende Material nicht unter der genannten Creative Commons Lizenz steht und die betreffende Handlung nicht nach gesetzlichen Vorschriften erlaubt ist, ist für die oben aufgeführten Weiterverwendungen des Materials die Einwilligung des jeweiligen Rechteinhabers einzuholen. 